\title{
Avaliação de potenciais interações medicamentosas em pacientes da unidade de terapia intensiva de um hospital universitário
}

\author{
Evaluation of potential drug interactions in patients of the intensive therapy unit of \\ university hospital
}

\author{
Alice Madilza Palmeira Gomes ${ }^{1}$, Karoline Gomes Dias Bezerra², Fernando de Sousa Oliveira ${ }^{3 *}$ \\ ${ }^{1}$ Farmacêutica pela UFCG; ${ }^{2}$ Graduanda em Farmácia pela UFCG; \\ ${ }^{3}$ Doutor e docente do Centro de Educação e Saúde / UFCG.
}

\begin{abstract}
Resumo
Introdução: entende-se por interação medicamentosa a ação de um medicamento, alimento ou qualquer substância química sobre o efeito de outro medicamento gerando uma resposta farmacológica ou clínica. Associações de fármacos no tratamento de doenças crônicas são muito comuns, principalmente em pacientes de unidade de terapia intensiva, no entanto, podem causar algumas interações medicamentosas, as quais podem ser responsáveis por insucesso terapêutico e aumento dos custos hospitalares. Objetivo: analisar a ocorrência e o perfil de potenciais interações medicamentosas em pacientes da UTI do Hospital Universitário Alcides Carneiro em Campina Grande-PB. Metodologia: realizou-se um estudo transversal descritivo, com abordagem quantitativa e qualitativa, a partir de dados coletados de prescrições da UTI entre janeiro e junho de 2017, utilizando um formulário específico. Para identificação das potenciais interações utilizou-se o Drug-Reax System do software Micromedex Health Series. Resultados: dentre as 109 prescrições avaliadas, 69,7\% apresentaram alguma interação, totalizando 244 potenciais interações. Foram detectados em média 6,9 medicamentos/prescrição. A maioria das interações apresentou mecanismo de ação farmacodinâmico, grau de severidade maior, razoável documentação e início de ação não especificado. Todas as prescrições com mais de 10 fármacos apresentaram algum tipo de interação. Conclusão: o surgimento de interações em UTI possui alta prevalência, mas as associações são justificadas pelo seu risco/benefício, por isso devem ser avaliadas e monitoradas pelo farmacêutico e a equipe de saúde, com intuito de reduzir falhas terapêuticas e risco ao paciente, aperfeiçoando assim o manejo clínico.
\end{abstract}

Palavras-chave: Interações Medicamentosas. Unidade de Terapia Intensiva. Efeitos dos Fármacos.

\begin{abstract}
Introduction: drug interaction is the action of a drug, food or any chemical substance on the effect of another drug generating a pharmacological or clinical response. Associations of drugs in the treatment of chronic diseases are very common, especially in intensive care unit patients, however, they may cause some drug interactions, which are responsible for therapeutic failure and increased hospital costs. Objective: analyzing the occurrence and the profile of potential drug interactions in patients of the Hospital Universitário Alcides Carneiro in Campina Grande-PB. Methodology: a descriptive cross-sectional study was conducted with a quantitative and qualitative approach, based on data collected from ICU medical prescriptions between January and June 2017, using a specific form. The Drug-Reax System of the Micromedex" Health Series software was used to identify the potential interactions. Results: among the 109 prescriptions evaluated, $69.72 \%$ presented some interaction, totaling 244 potential interactions. 6.91 medications/prescription were detected on average. The majority of the interactions presented pharmacodynamic mechanism of action, greater degree of severity, reasonable documentation and unspecified onset of action. All prescriptions with more than 10 drugs presented some type of interaction. Conclusion: the emergence of interactions in ICUs has high prevalence, but the associations are justified by their risk I benefit, so they should be evaluated and monitored by the pharmacist and the health team, in order to reduce the emergence of potential interactions that lead to therapeutic failure and risk to the patient, thus improving clinical management.
\end{abstract}

Keywords: Drug Interactions. Intensive Care Units. Drug Effects.

\section{INTRODUÇÃO}

Na prática clínica é comum o uso simultâneo de diversos medicamentos, aumentando assim a ocorrência de interações medicamentosas (IMs). A politerapia é uma estratégia útil para obter o efeito terapêutico sinergístico

Correspondente/ Corresponding: *Fernando de Sousa Oliveira - Centro de Educação e Saúde / Universidade Federal de Campina Grande, acesso Profa. Maria Anita Furtado Coelho s/n, Olho D'Água da Bica, Cuité-PB, CEP: 58175-000 - Tel.: (83)3372-1900 - E-mail: fernandoufcg@ hotmail.com ou para a terapia de múltiplas doenças coexistentes. No entanto, contribui significativamente para o desenvolvimento de reações adversas e IMs, causando problemas à saúde do paciente, prologando assim, o tempo de internação e aumentando os custos dos hospitais ${ }^{1,2}$.

Pode-se definir IM como um evento que ocorre quando um fármaco é administrado concomitantemente com outro fármaco ou alimento, e que esse tem seus efeitos modificados pela ação do outro, podendo resultar em diminuição, anulação ou aumento do efeito de um deles ${ }^{3}$.

Há fatores de risco que favorecem a ocorrência das 
IMs, os quais estão relacionados ao medicamento, ao paciente e à prescrição medicamentosa. Com relação aos fatores relacionados ao medicamento, o principal é a potência em induzir ou inibir o sistema enzimático de biotransformação de um fármaco, influenciando na margem terapêutica, a qual representa a relação existente entre a dose máxima tolerada e a dose terapêutica mínima. Dentre os principais fatores de risco relacionados ao paciente estão as características de alguns grupos mais vulneráveis, são eles: idosos, pacientes imunodeprimidos, submetidos a cirurgias e internos em terapia intensiva. Quanto aos fatores relacionados à prescrição, destacam-se os múltiplos medicamentos presentes na prescrição associado ao complicado estado clínico dos pacientes, em âmbito hospitalar. Além disso, o agravamento das IMs pode ocorrer pela falta de conhecimento dos profissionais sobre a ação dos fármacos ${ }^{4}$.

Associar episódios clínicos com IMs é um trabalho difícil de documentar, isso faz com que se busque, cada vez mais, avaliar prescrições de medicamentos. Com isso, o profissional farmacêutico é importante no sentido de monitorar os medicamentos administrados aos pacientes, revisar prescrições e protocolos de medicamentos prescritos, assim como, garantir efetividade e segurança da farmacoterapia, objetivando ainda reduzir custos e oferecer um adequado serviço no âmbito hospitalar ${ }^{2,5,6}$.

A incidência de IMs em hospitais varia de 3 a $5 \%$ em pacientes que fazem uso de até quatro medicamentos, esse número aumenta para $20 \%$ ou mais, para aqueles que fazem uso de 10 a 20 medicamentos, evidenciando assim a importância e significância desse problema ${ }^{7}$.

A unidade de terapia intensiva (UTI) é o setor do hospital caracterizado pela complexidade do atendimento ao paciente em estado crítico, sendo estes fortes candidatos a apresentar IMs, podendo contribuir para o insucesso terapêutico e provocar sérios danos ao paciente ${ }^{4}$.

Há estudos que mostram as IMs sendo bem mais frequentes em pacientes de UTI, quando comparados a pacientes de outras unidades hospitalares ${ }^{8}$. Os estudos sobre IMs no âmbito da terapia intensiva são escassos e a maioria limitados a quantificação dessas IMs.

Desta forma, o presente estudo teve como objetivo analisar o perfil e a ocorrência de potenciais IMs em prescrições de pacientes internados na UTI do Hospital Universitário Alcides Carneiro (HUAC) em Campina Grande-PB, visando contribuir para o conhecimento sobre o assunto e auxiliar os profissionais de saúde sobre IMs a partir das informações geradas nesse estudo, proporcionando assim, uma escolha adequada da farmacoterapia que deverá ser utilizada por cada paciente, garantindo um tratamento seguro e efetivo.

\section{METODOLOGIA}

Trata-se de um estudo transversal, descritivo, com abordagem quantitativa e qualitativa, realizado na Farmácia Central do HUAC, a partir da análise das prescrições da UTI - Adulto, durante o período de janeiro a junho de 2017. Para análise das potenciais IMs, foi utilizada a primeira prescrição de cada paciente maior de 18 anos de idade que ingressou na UTI, com mais de um medicamento na prescrição e permanecendo internado por um período de 24 horas ou mais.

$\mathrm{Na}$ análise quantitativa foram observadas as seguintes variáveis: número de medicamento por prescrição, número de IM por prescrição, fármacos mais prescritos, fármacos mais envolvidos em IMs, relação entre número de fármacos e interações, relação entre sexo e interações, número de interações farmacocinéticas e farmacodinâmicas.

As potencias IMs foram classificadas quanto ao mecanismo de ação (farmacocinético, farmacodinâmico e desconhecido), o grau de severidade (maior, moderado, menor e contraindicado), o início de ação (não especificado, tardio, rápido) e o grau de embasamento na literatura científica (excelente, bom e razoável).

Para identificação e classificação das IMs foi utilizado o DrugReax System, um dos sistemas do programa MICROMEDEX Health Series, com acesso em rede por meio do portal de periódicos da CAPES.

Os valores obtidos nesse estudo foram armazenados no programa Microsoft Office Excel ${ }^{\circ} 2010$, para produção dos gráficos e tabelas correspondentes, os quais foram posteriormente analisados e interpretados.

O estudo foi conduzindo de acordo com as normas e diretrizes da Resolução № 466/2012 do Conselho Nacional de Saúde e aprovado pelo Comitê de Ética em Pesquisa Envolvendo Seres Humanos, sob parecer no 1.612.257.

\section{RESULTADOS E DISCUSSÃO}

Durante o período do estudo foram analisadas 109 prescrições, destas, $76(69,7 \%)$ apresentaram potencial para algum tipo de IM. Esse valor obtido está em conformidade com os percentuais encontrados em outros estudos semelhantes cuja variação foi de $49,7 \%$ a $88,2 \%{ }^{9}$ Dos 109 pacientes que constituíam a amostra, 55 (50,5\%) eram do gênero feminino, destes, $35(63,6 \%)$ tinham prescrições que apresentaram algum tipo de interação. Dentre os 54 (49,5\%) pacientes do gênero masculino, 41 $(75,9 \%)$ tinham prescrição com alguma IM.

Percebe-se que a prevalência de IMs é maior em pacientes do sexo masculino, isso pode ser justificado pela resistência dos homens na procura por serviços de saúde. Segundo a Política Nacional de Atenção Integral à Saúde do Homem (PNAISH), o homem se define como um ser invulnerável, o que acaba influenciando para a negligência do autocuidado, além de se exporem a maiores situações de risco, tais como o uso de álcool, e serem mais propensos a adquirir doenças, sobretudo as graves e crônicas ${ }^{1,10}$. Em consequência disso, quando o homem vem a ser hospitalizado, utiliza uma grande quantidade de medicamentos e, segundo a literatura, quanto maior o número de medicamentos utilizados, mais frequente e 
maior o tempo de internação, aumentando assim o risco de ocorrência de $\mathrm{IMs}^{11}$.

Durante o estudo, verificou-se a presença de 244 possíveis IMs, sendo 117 diferentes umas das outras, em uma média de 2,24 interações por prescrição. A média de fármacos correspondeu a 6,91 fármacos/prescrição.

Nas prescrições com até cinco medicamentos, $42,5 \%$ apresentaram pelo menos um tipo de interação. No entanto, as que possuíam de 6 a 10 fármacos prescritos, $81,5 \%$ delas apresentaram IM; para aquelas que possuíam mais de 10 medicamentos, todas apresentaram IM (tabela 1). Percebe-se então que existe uma relação entre o número de fármacos prescritos e a presença de IMs, sendo essa relação considerada como um importante fator de risco para a ocorrência dessas potenciais interações ${ }^{9}$.

Tabela 1 - Relação entre o número de fármacos prescritos e prevalência de possíveis interações medicamentosas.

\begin{tabular}{lccc}
\hline $\begin{array}{c}\text { Número de } \\
\text { fármacos } \\
\text { prescritos }\end{array}$ & $\begin{array}{c}\text { Quantidade } \\
\text { prescrições }\end{array}$ & $\begin{array}{c}\text { Quantidade de } \\
\text { prescrições com } \\
\text { interações }\end{array}$ & $\begin{array}{c}\text { Porcentagem de } \\
\text { prescrições com } \\
\text { interação }\end{array}$ \\
\hline Até 5 & 40 & 17 & 42,5 \\
Entre 6-10 & 54 & 44 & 81,5 \\
Mais de 10 & 15 & 15 & 100 \\
\hline
\end{tabular}

Fonte: Dados da pesquisa

Geralmente, a incidência de IMs em UTI é bem maior que nas demais alas do ambiente hospitalar, isso se justifica pelo fato dos pacientes fazem uso de múltiplos medicamentos, os quais, na maioria das vezes, são administrados simultaneamente. Há também o risco atribuído à gravidade das doenças e à falência dos órgãos ${ }^{12}$.

Nas 109 prescrições foram encontrados 114 diferentes fármacos prescritos, sendo o omeprazol (inibidor da bomba de prótons) o mais prescrito em 66 prescrições $(60,6 \%)$; seguido da enoxaparina (antitrombótico) em 45 prescrições $(41,3 \%)$; dipirona (analgésico central não opioide), em 43 (39,4\%); e a ondansetrona (antiemético) em $40(36,7 \%)$ das prescrições avaliadas (tabela 2). Resultados semelhantes foram observados em dois estudos, com os fármacos dipirona, enoxaparina, furosemida, omeprazol e fentanil também estando entre os mais frequentes nas prescrições ${ }^{1,13}$.

A dipirona está incluída na lista dos medicamentos mais prescritos. No entanto, este medicamento não se encontrava disponível na base de dados do Micromedex ${ }^{\circ}$ por ser uma substância proscrita nos Estados Unidos. Mesmo tendo sido o terceiro medicamento mais prescrito do estudo, não foi incluído na busca das interações.
Tabela 2 - Fármacos mais prescritos na UTI-Adulto do HUAC.

\begin{tabular}{lcc}
\hline Fármacos & $\begin{array}{c}\text { Número de } \\
\text { prescrições }\end{array}$ & $\begin{array}{c}\text { Porcentagem de } \\
\text { prescrições }\end{array}$ \\
\hline Omeprazol & 66 & 60,6 \\
Enoxaparina & 45 & 41,3 \\
Dipirona & 43 & 39,4 \\
Ondansetrona & 40 & 36,7 \\
KCl & 34 & 31,2 \\
Midazolam & 33 & 30,3 \\
Fentanil & 31 & 28,4 \\
Furosemida & 22 & 20,2 \\
Meropeném & 22 & 20,2 \\
Ciprofloxacino & 17 & 15,6 \\
\hline
\end{tabular}

Fonte: Dados da pesquisa

Dentre as 76 prescrições que apresentaram algum tipo de interação, o fármaco que mais se destacou interagindo com o maior número de outros fármacos foi o fentanil, o qual interagiu com 13 fármacos diferentes $(20,9 \%)$, dentre os principais: midazolam, ciprofloxacino, fenobarbital, ranitidina e tramadol. 0 ácido acetilsalicílico (AAS) participou de 12 interações e a ondansetrona de 10 (figura 1).

Segundo um estudo realizado na UTI de um hospital público em Feira de Santana-BA, o AAS, o midazolam e o fentanil também foram os fármacos que mais se envolveram em $\mathrm{IMs}^{1}$.

Figura 1 - Fármacos envolvidos em interações medicamentosas.

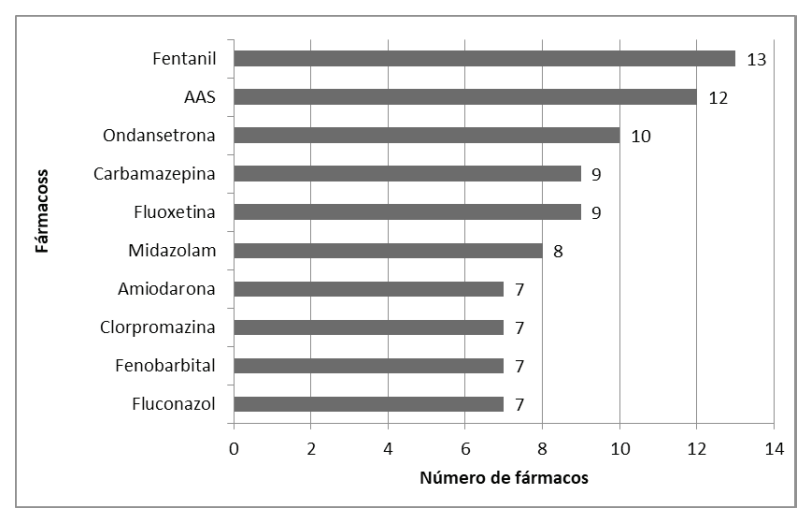

Fonte: Autoria própria

Entre as 76 prescrições que apresentam IMs, destacaram-se as 10 interações mais prevalentes e sua classificação quanto ao mecanismo de ação, severidade, início de ação e documentação (tabela 3). Estas interações corresponderam a $43,8 \%$ de todas as interações encontradas no presente estudo. 
Tabela 3 - Interações medicamentosas mais prevalentes e sua classificação.

\begin{tabular}{|c|c|c|c|c|}
\hline Interação & Mecanismo de ação & Severidade & Início de ação & $\begin{array}{l}\text { Embasamento na } \\
\text { literatura }\end{array}$ \\
\hline Fentanil + midazolam & Farmacodinâmico: aumento do risco de depressão do SNC & Maior & Não especificado & Razoável \\
\hline Midazolam + omeprazol & Farmacocinético: retardo do metabolismo do midazolam & Moderado & Tardio & Razoável \\
\hline Ondansetrona + tramadol & Desconhecido: redução da eficácia do tramadol & Moderado & Não especificado & Excelente \\
\hline Ciprofloxacino + metronidazol & Farmacodinâmico: efeitos aditivos sobre o intervalo QT & Maior & Não especificado & Razoável \\
\hline Metronidazol + ondansetrona & Farmacodinâmico: efeitos aditivos sobre o intervalo QT & Maior & Não especificado & Razoável \\
\hline AAS + enoxaparina & $\begin{array}{l}\text { Farmacocinético: diminuição da função plaquetária e da } \\
\text { coagulação }\end{array}$ & Maior & Não especificado & Boa \\
\hline Ciprofloxacino + ondansetrona & Farmacodinâmico: efeitos aditivos sobre o intervalo QT & Maior & Não especificado & Razoável \\
\hline Ciprofloxacino + fentanil & Farmacocinético: inibição do metabolismo do fentanil & Maior & Não especificado & Razoável \\
\hline Enoxaparina + fluoxetina & Desconhecido: aumento do risco de hemorragia & Maior & Tardio & Boa \\
\hline Furosemida + hidrocortisona & Desconhecido: reducão dos níveis de $\mathrm{K}^{+}$ & Moderado & Tardio & Razoável \\
\hline
\end{tabular}

Fonte: Dados da pesquisa

Delineando o perfil das IMs mais prevalentes, foi observado que estas possuem, em sua maioria, mecanismo de ação farmacodinâmico, gravidade maior, início de ação não especificado e razoável documentação. É de suma importância conhecer o mecanismo de ação dos fármacos por toda a equipe de saúde para que se compreendam os mecanismos farmacológicos envolvidos nas IMs, pois assim pode ser possível impedir o seu surgimento ou diminuir os danos através de um planejamento do manejo clínico. Quando à gravidade da IM é classificada como maior, demonstra-se que estas interações têm relevada importância clínica. Com relação ao início de ação não especificado, faz-se necessário investigar as possíveis IMs, pois pode ser que os sinais e sintomas da interação surjam apenas após a alta hospitalar, sendo essa uma das maiores preocupações com relação ao efeito da interação. Essa situação nos remete o quão importante é o processo de reconciliação de medicamentos para a segurança do paciente em alta da UTI. A documentação razoável afirma a necessidade de mais estudos no que se refere às IMs e que estes sejam bem monitorados ${ }^{14}$.

A interação mais prevalente foi entre fentanil e midazolam, assim como em outros estudos. Essa IM possui mecanismo de ação farmacodinâmico, gravidade maior, início de ação não especificado e razoável documentação. A principal consequência dessa interação é a depressão respiratória, seguida pela sedação excessiva. Considera-se como uma interação farmacodinâmica com base no sinergismo farmacológico, isto é, quando o efeito resultante de dois fármacos é superior à soma dos efeitos de cada fármaco separadamente. Devido ao tempo de início de ação desta interação ser não especificado, é recomendado monitorização contínua do paciente, redução da dose de um dos fármacos, ou de ambos, para que os efeitos dessa associação sejam amenizados ${ }^{1}$.

Em UTI, a associação de fentanil e midazolam é utilizada com finalidade terapêutica, pois fornece conforto e redução da ansiedade de pacientes em ventilação me- cânica, como também para melhora da oxigenação. 0 adequado uso destes fármacos na sedação pode melhorar o tempo do paciente na ventilação mecânica. $O$ desafio, entretanto, é garantir o grau adequado de sedação, pois, caso contrário, isso pode resultar em agitação, delirium e prolongamento do uso do ventilador mecânico ${ }^{9,14,15}$.

Dentre as interações encontradas, observou-se que $113(46,3 \%)$ apresentavam mecanismo de ação farmacodinâmico, $87(35,7 \%)$ possuíam mecanismo do tipo farmacocinético e $44(18,0 \%)$ possuíam mecanismo de ação desconhecido (figura 2).

Figura 2 - Mecanismos de ação envolvidos nas potenciais interações medicamentosas.

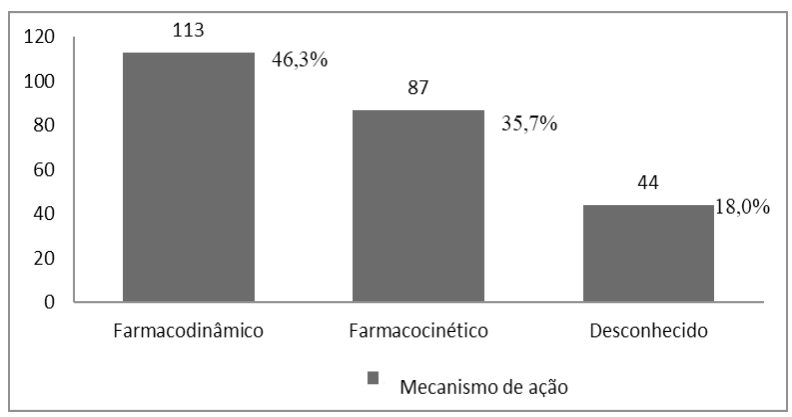

Fonte: Fonte: Autoria própria

As interações farmacodinâmicas ocorrem quando os efeitos de um fármaco são alterados pela presença de outro no seu sítio de ação ou no mesmo sistema fisiológico, enquanto que, as interações farmacocinéticas acontecem quando um fármaco modifica os processos de absorção, distribuição, biotransformação ou excreção de outro fármaco ${ }^{16}$.

A associação de enalapril e furosemida é um exemplo de interação farmacodinâmica. O primeiro fármaco age inibindo a enzima conversora de angiotensina (ECA), bloqueando a biotransformação da angiotensina I em II no sangue e nos tecidos, levando à vasodilatação e diminui- 
ção da resistência vascular periférica. Além disso, inibe a degradação da bradicinina, que atua como vasodilatador. A furosemida, por ser é um potente diurético com ação no ramo espesso ascendente da alça de Henle, promove uma relativa depleção de volume intravascular, assim a ocorrência clínica mais importante proveniente desta associação é a hipotensão, devido ao efeito aditivo. Isso justifica a utilização deste sinergismo para fins terapêuticos. No entanto, é importante monitorar a hipotensão, o estado líquido e o peso corporal do paciente regularmente por até duas semanas, após o ajuste de dose ${ }^{15,17}$.

As interações do tipo farmacocinéticas estão em segundo lugar dentre as encontradas no estudo. A associação de midazolam e omeprazol foi a interação de mecanismo farmacocinético mais prevalente no estudo, mesmo resultado encontrado por uma pesquisa desenvolvida em UTIs de sete hospitais de ensino no Brasil ${ }^{12}$. Os principais efeitos desta interação são depressão no SNC, ataxia e letargia. Acredita-se que o omeprazol, por ser um inibidor do conjunto de enzimas que no processo de metabolização promovem a oxidação do midazolam (CYP3A4), reduza a biotransformação desse fármaco, atrasando assim sua eliminação, resultando em efeitos mais intensos e prolongados. É sugerido que se utilize outros fármacos que possuam o mesmo efeito terapêuti$\mathrm{co}$, mas que não desenvolva essa IM, como por exemplo, o pantoprazol, fármaco que atua diminuindo a acidez gástrica pelo mesmo mecanismo de ação, mas que não inibe o CYP3A4, evitando assim essa interação metabólica. Em casos de utilização da associação do omeprazol e midazolam, é importante um monitoramento constate dos pacientes, principalmente quanto ao nível de sedação e, além disso, deve ser feito o ajuste de dose quando necessário. Um exemplo de IM com mecanismo de ação desconhecido ocorre entre ondansetrona e tramadol. Tal associação pode causar redução da eficácia do tramadol. Essa diminuição da eficácia deve ser monitorada, quando o mesmo estiver associado com a ondansetrona ${ }^{15}$.

Quanto ao grau de severidade, a grande maioria das IMs, que corresponde a $136(55,7 \%)$ interações, classificam-se como de severidade maior e $97(39,8 \%)$ delas são de severidade moderada (figura 3). Em um estudo semelhante, realizado no Hospital Israelita Albert Einstein, a maioria das interações $(33,9 \%)$ apresentaram severidade maior, seguida das interações de severidade moderada $(27,2 \%)^{18}$.

Diante da prevalência de IMs de maior severidade, nota-se a importância do diagnóstico precoce desse tipo de interação, no momento da análise da prescrição, podendo assim, prevenir a ocorrência de eventos adversos, pois a gravidade da interação é o fator de determina ao profissional de saúde, a necessidade de alguma intervenção.

As interações consideradas de severidade maior podem ser definidas como aquelas que oferecem risco de morte ao paciente e/ou necessitam de intervenção imediata. Dentre elas, pode-se citar a associação entre ciprofloxacino e metronidazol, a qual representa a quarta interação mais prevalente no estudo. Esta possui maior severidade, pois o uso de metronidazol com fármacos que prolongam o intervalo QT pode aumentar o risco de efeitos cardiovasculares graves e torsades de points. A associação de amitriptilina e haloperidol e da clorpromazina com risperidona podem gerar os mesmos efeitos citados anteriormente, sendo necessário o monitoramento do eletrocardiograma no início e durante a terapêutica concomitante ${ }^{4,9,15}$.

Figura 3 - Classificação das interações medicamentosas quanto ao grau de severidade.

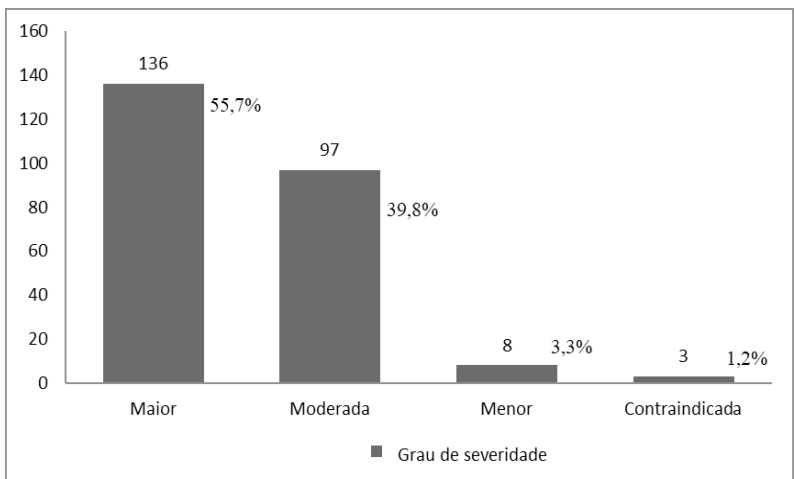

Fonte: Autoria própria

Vale salientar que diversas IMs são subdiagnosticadas e que algumas, mesmo que fatais, não são reconhecidas, o que reforça ainda mais a necessidade de estudos sobre mecanismo de ação dos fármacos, possíveis IMs e pesquisas nessa área. $O$ farmacêutico, por ser o profissional mais ligado ao estudo de fármacos, deve buscar informações e traçar estratégias que abranjam a equipe multidisciplinar de saúde para que estes seja capacitados a prevenir, identificar e resolver a problemática das IMs, com intuito de minimizar estas interações, principalmente as de severidade maior ${ }^{5}$.

São consideradas interações de moderada severidade, aquelas que podem resultar em exacerbação das condições clínicas e/ou necessitarem de mudança na terapia. Temos como exemplo, a interação entre furosemida e hidrocortisona, a qual pode resultar em hipocalemia. Os níveis séricos de potássio devem ser cuidadosamente monitorados ${ }^{15}$.

As interações de severidade menor possuem efeitos clínicos limitados, sem requerer mudanças na terapia. A interação AAS e ranitidina é um exemplo desse tipo de IM. O uso simultâneo desses fármacos pode ocasionar redução dos níveis sanguíneos do AAS, com consequente redução do seu efeito antiplaquetário. Estudos sugerem que o mecanismo de ação pelo qual essa interação ocorre seja pela alteração na absorção do AAS. Podem ser classificadas como de severidade contraindicada, as interações nas quais os fármacos envolvidos não podem ser utilizados simultaneamente. Um exemplo é a interação entre 
clorpromazina e metoclopramida. A metoclopramida é utilizada como antiemético, possui estrutura química semelhante à clorpromazina e atua como antagonista $\mathrm{D}_{2}$, podendo induzir a quadro de parkinsonismo. $\mathrm{O}$ uso de metoclopramida e antipsicóticos pode causar um aumento do risco de reações extrapiramidais ou síndrome maligna dos neurolépticos. A interação é considerada contraindicada por seus riscos, apesar dos efeitos da interação serem pouco conhecidos ${ }^{15}$.

No que se refere ao início de ação, 149 (61,1\%) das interações encontradas apresentaram início de ação não especificado, $70(28,7 \%)$ de início tardio e $5(10,2 \%)$ interações foram classificadas com início de ação rápido (figura 4).

Em um estudo transversal e descritivo realizado em três UTIs do Hospital Universitário da Universidade Estadual de Londrina-PR, foi possível observar que $34,4 \%$ das interações apresentaram início de ação não especificado, um número bem menor quando relacionado com o deste trabalho, enquanto que $42,8 \%$ possuíam início de ação tardio e apenas $22,8 \%$, início de ação rápido ${ }^{19}$.

Figura 4 - Classificação das interações medicamentosas quanto ao início de ação.

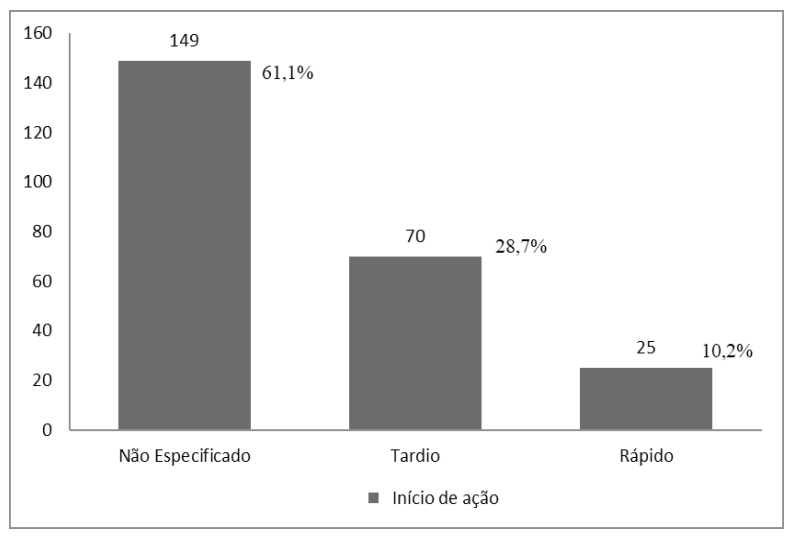

Fonte: Autoria própria

As interações classificadas como de início de ação rápido, são aquelas em que os efeitos adversos tornam-se evidentes nas 24 horas após a administração dos fármacos; as de início tardio, os efeitos tornam-se evidentes dias ou semanas após a administração farmacológica; e as IM com início não especificado, ocorrem quando não apresentam o tempo de início dos eventos descritos na literatura ${ }^{3}$.

Dentre as interações de início de ação não especificado está a que ocorre entre AAS e enoxaparina. A literatura afirma que o uso de heparinas de baixo peso molecular e anticoagulantes simultaneamente, aumenta o risco de sangramento no paciente. Assim, quando tais fármacos são utilizados de modo concomitante, deve-se monitorar o paciente em razão do risco de sangramento, sobretudo sangramento gastrintestina $\left.\right|^{15}$.

Um exemplo de interação com início de ação tardio ocorre entre a levotiroxina e omeprazol. É uma interação que resulta no aumento dos níveis de TSH, no entanto, seu mecanismo de ação é desconhecido. Interação de início rápido foi observada na associação entre amiodarona e fentanil. Essa IM possui mecanismo de ação farmacocinético, no qual a amiodarona inibe o metabolismo do fentanil através do CYP4503A4, podendo causar cardiotoxicidade e aumento dos efeitos tóxicos do fentanil ${ }^{15}$.

Quanto à documentação, as interações classificam-se em excelente, boa e razoável. Das interações encontradas, observou-se que mais da metade, um total de 156 IMs $(63,9 \%)$ apresentavam documentação razoável, 60 $(24,6 \%)$ eram de documentação boa e apenas $28(11,5 \%)$ interações apresentavam documentação excelente.

Em um estudo quantitativo desenvolvido na UTI de dois hospitais de Goiânia-GO, o resultado difere do encontrado no presente estudo com relação às interações classificadas como de boa documentação $(57,5 \%)$ e uma menor parcela $(11,2 \%)$ apresenta documentação excelente, sendo este último valor bem semelhante do encontrado no presente estudo ${ }^{14}$.

As interações são classificadas como de excelente documentação, quando existem diversos ensaios clínicos controlados comprovando a sua existência. Dentre as IMs que são consideradas de excelente documentação, encontra-se a que ocorre entre AAS e enalapril. Tal associação ocasiona uma diminuição na eficácia do enalapril. As classificadas como de boa documentação são as interações que possuem documentação que sugerem sua existência, no entanto, faltam ensaios clínicos que a comprovem. Nesse caso, pode-se citar a associação de diazepam e omeprazol. O omeprazol reduz o metabolismo do diazepam e atrasa a sua eliminação, aumentando os níveis séricos do benzodiazepínico, resultando em acentuados efeitos de sedação e ataxia ${ }^{15}$.

As interações que são de documentação razoável são as que possuem pobre documentação, mas existem considerações farmacológicas para a sua existência, como ocorre com metoclopramida e tramadol. Esta associação pode potencializar os efeitos sedativos do tramadol e causar outros efeitos indesejáveis, como o aumento de risco de convulsões ${ }^{20}$.

É importante ressaltar a contribuição expressiva do conhecimento do perfil e ocorrência de IMs em UTIs, que podem servir de instrumento para que ações, visando a melhoria da segurança do paciente, sejam planejadas. $O$ programa utilizado apresenta alta sensibilidade e adequada especificidade para identificar potenciais interações, isso contribui positivamente na validade dos resultados encontrados.

Neste sentido, é notória a importância da inserção do profissional farmacêutico na equipe multidisciplinar, como também, a presença do farmacêutico clínico na UTI. Este profissional pode contribuir de forma significativa na segurança e eficácia da terapia do paciente crítico, avaliando as prescrições e identificando possíveis IMs. O farmacêutico irá prevenir a ocorrência desses eventos adversos, estimando o risco/benefício para decidir sobre a 
manutenção ou mudança na terapia prescrita. Além disso, pode auxiliar continuamente na educação em saúde e na redução dos riscos derivados da farmacoterapia.

\section{CONCLUSÃO}

Diante dos resultados obtidos, observou-se que uma considerável parcela da amostra deste estudo apresentou IMs, levando a afirmar a alta prevalência de interações em UTIs. Pode-se perceber que quanto maior o número de medicamentos por prescrição, maior o número de interações. Alguns dos medicamentos que estão entre os mais prescritos, também figuram entre os que mais se envolveram em IMs.

Esta informação é útil para que os profissionais de saúde, principalmente os que atuam na UTI, fiquem atentos e dediquem uma maior atenção, quando estes medicamentos estiverem presentes nas prescrições. A maioria das interações apresentou mecanismo de ação farmacodinâmico, grau de severidade maior, início de ação não especificado e razoável documentação.

Para reduzir o surgimento de possíveis interações é indispensável à monitorização das prescrições, sendo necessário que os profissionais de saúde detenham maior conhecimento a cerca dos mecanismos de ação das potenciais interações. Isso ressalta ainda mais a importância do profissional farmacêutico atuando na equipe multidisciplinar, avaliando as prescrições, intervindo junto aos prescritores, quando necessário, e prevenindo interações antes da dispensação, o que ameniza os riscos provenientes da terapia farmacológica.

\section{REFERENCIAS}

1. CEDRAZ, K. N.; SANTOS JUNIOR, M. C. Identificação e caracterização de interações medicamentosas em prescrições médicas da unidade de terapia intensiva de um hospital público da cidade de Feira de Santana, BA. Rev. Soc. Bras. Clín. Méd., São Paulo, v. 12, n. 2, p. 1-7, 2014.

2. SOUSA, A. L. B. et al. Atenção farmacêutica humanizada em pacientes hipertensos no Hospital Universitário Lauro Wanderley. Rev. Ciênc. Méd. Biol., Salvador, v. 16, n. 1, p. 45-51, 2017.

3. LIMA, R. E. F.; CASSIANI, S. H. B. Potential drug interactions in intensive care patients at a teaching hospital. Rev. Latinoam. Enferm., Ribeirão Preto, v. 17, n. 2, p. 222-227, 2009.

4. FARIA, L. M. P.; CASSIANI, S. H. B. Interação medicamentosa: conhecimento de enfermeiros das unidades de terapia intensiva. Acta Paul. Enferm., São Paulo, v. 24, n. 2, p. 264-70, 2011.

5. CARDINAL, L. S. M. et al. Caracterização das prescrições medicamentosas em unidade de terapia intensiva adulto. Rev. Bras. Ter. Intensiva, São Paulo, v. 24, n. 2, p. 151-156, 2012.

6. MOURA, C. S.; TAVARES, L. S.; ACURCIO, F. A. Interação medicamentosa associada à reinternação hospitalar: estudo retrospectivo em um hospital geral. Rev. Saúde Pública, São Paulo, v. 46, n. 6, p. 1082-1089, 2012.

7. AMARAL, D. M. D.; PERASSOLO, M. S. Possíveis interações medicamentosas entre os anti-hipertensivos e antidiabéticos em participantes do Grupo HIPERDIA de Parobé, RS (Uma análise teórica). Rev. Ciênc. Farm. Básica Apl., Araraquara, v. 33, n. 1, p. 99-105, 2012.

8. MIBIELLI, P. et al. Interações medicamentosas potenciais entre idosos em uso dos anti-hipertensivos da Relação Nacional de Medicamentos Essenciais do Ministério da Saúde do Brasil. Cad. Saúde Pública, Rio de Janeiro, v. 30, n. 9, p. 1947-1956, 2014.

9. ROCHA, P. C. F.; MOTA, P. S.; OLIVEIRA, C. I. F. B. Prevalência de potenciais interações medicamentosas em uma unidade de terapia intensiva de Manaus-AM. Rev. Bras. Farm., Rio de Janeiro, v. 95, n. 3, p. 909-923, 2014.

10. BRASIL. Ministério da Saúde. Política nacional de atenção integral à saúde do homem. Secretaria de atenção à saúde. Departamento de ações programáticas estratégicas. Brasília, DF, 2008.

11. VIEIRA, L. B. et al. Interações Medicamentosas Potenciais em Pacientes de Unidades de Terapia Intensiva. Rev. Ciênc. Farm. Básica Apl., Araraquara, v. 33, n. 3, p. 401-408, 2012.

12. CARVALHO, R. E. F. L. et al. Prevalência de interações medicamentosas em unidades de terapia intensiva no Brasil. Acta Paul. Enferm., São Paulo, v. 26, n. 2, p. 150-7, 2013.

13. NÓBREGA, R. C.; BATISTA, L. M.; RIBEIRO, N. K. R. Perfil de utilização de anti-infecciosos e interações medicamentosas potenciais em unidade de terapia intensiva. Rev. Bras. Farm. Hosp. Serv. Saúde, São Paulo, v. 3, n. 3, p. 28-32, 2012

14. POMBO-NASCIMENTO, E. et al. Avaliação de prescrições quanto à ocorrência de possíveis interações medicamentosas na Clínica Médica do Hospital Geral de Bonsucesso. Rev. Bras. Farm., Rio de Janeiro, v. 88, n. 1, p. 14-16, 2007.

15. MICROMEDEX ${ }^{\circ}$ HEALTHCARE SERIES. Base de Dados. Disponível em: <https://www.thomsonhc.com/hcs/librarian/CS/70A4EC/PFActionld/ pf.HomePage/ssl/true>. Acesso em: 05 abr. 2019.

16. VASCONCELOS, D. I. B. et al. Investigação do perfil da utilização de medicamentos e interações medicamentosas em anciãos de Petrópolis -RJ. Rev. Eletrônica Estácio Saúde, Petrópolis, v. 4, n. 1, p. 27-34, 2015.

17. MARQUITO, A. B. et al. Interações medicamentosas potenciais em pacientes com doença renal crônica. J. Bras. Nefrol., São Paulo, v. 36, n. 1, p. 26-34, 2014.

18. ALMEIDA, S. M.; GAMA, C. S.; AKAMINE, N. Prevalência e classificação de interações entre medicamentos dispensados para pacientes em terapia intensiva. Einstein, São Paulo, v. 5, n. 4, p. 347-51, 2007.

19. OLIVEIRA-PAULA, G. H. et al. Interações medicamentosas potencias em unidades de terapia intensiva de um hospital do Sul do Brasil. Semina Ciênc. Biol. Saúde, Londrina, v. 35, n. 2, p. 21-30, 2014.

20. PINTO, L. H.; SOUZA, H.; CARNEIRO, T. K. Avaliação da frequência de interações medicamentosas ocorridas com pacientes internados em clínica cirúrgica em um hospital público de Joinville. Rev. Eletrônica Farm., Goiânia, v. 12, n. 2, p. 16-29, 2015.
Submetido em: 30/01/2019

Aceito em: 15/04/2019 